CASE REPORT

\author{
K.F. Layton \\ J.H. Hise \\ I.C. Thacker
}

\section{Recurrent Intracranial Stenosis Induced by the Wingspan Stent: Comparison with Balloon Angioplasty Alone in a Single Patient}

\begin{abstract}
SUMMARY: We present a case in which angioplasty alone and stent-assisted angioplasty were performed in the same patient to treat medically refractory intracranial stenoses. This elderly patient with presumed intracranial atherosclerotic disease underwent angioplasty alone for his anterior cerebral artery stenosis. Stent-assisted angioplasty was used for treatment of his ipsilateral middle cerebral artery stenosis. Follow-up angiography at 4 months documented severe recurrent stenosis confined only to the stented portion of the middle cerebral artery.
\end{abstract}

T he risk of ischemic stroke in patients with intracranial atherosclerotic disease (ICAD) ranges from $8 \%$ to $22 \% .^{1,2}$ Despite receiving maximum medical therapy, many patients with ICAD experience recurrent transient ischemic attacks (TIAs) or stroke. Due to the poor surgical results for intracranial bypass procedures, physicians have been searching for other methods to reduce the incidence of TIA and stroke in patients with ICAD. ${ }^{2}$

Recently, endovascular techniques have been used to treat stenoses related to ICAD in an effort to reduce the occurrence of TIA and stroke. Historically, the results of angioplasty alone have been promising. ${ }^{3}$ Advances in balloon engineering have allowed for easier access to smaller and more tortuous intracranial vessels. Intracranial stent placement has been traditionally reserved as a rescue measure during balloon angioplasty. Flow-limiting dissections, abrupt vessel occlusion, and recalcitrant high-grade stenoses were the most common indications for intracranial stent placement after angioplasty. Before the development of the Wingspan stent (Boston Scientific, Natick, Mass), stents designed for the coronary vasculature were the only available tools. These stents were often suboptimal due to their relatively inflexible design. The tortuous cerebral vasculature often posed a significant hurdle to coronary stent navigation. With the recent introduction of the self-expanding Wingspan stent (Boston Scientific), it is now much easier to deliver a stent through difficult cerebral arterial anatomy.

Despite the obvious advantages of this new device, appropriate indications for its use are still unclear. There are currently no randomized, controlled trials comparing intracranial stent-assisted angioplasty with angioplasty alone or traditional medical therapy. Results from recent Wingspan stent registry data have raised some significant concerns over the unfettered use of this device in patients with intracranial arterial stenoses. ${ }^{4,5}$

We present a patient with ICAD who underwent angioplasty alone and stent-assisted angioplasty in 2 adjacent intracranial arteries. Follow-up angiography at 4 months demonstrated the vessel treated with angioplasty alone to have a

Received November 16, 2007; accepted after revision January 2, 2008.

From the Department of Radiology, Baylor University Medical Center, Dallas, Tex.

Please address correspondence to Kennith F. Layton, Department of Radiology, Baylor University Medical Center, 3500 Gaston Ave, Dallas, TX 75246; e-mail: klayton@americanrad.com

DOI 10.3174/ajnr.A1006 normal caliber. However, the vessel receiving stent implantation developed a severe in-stent restenosis. Because the 2 vessels were in close proximity and obviously related to the same underlying etiology, the recurrent in-stent stenosis was probably caused by the stent. Clinical history, procedural details, and current topics related to intracranial stent implantation are discussed.

\section{Case Report}

\section{Clinical Information}

A 68-year-old white man was transferred from an outside hospital for evaluation of a recent ischemic stroke. He presented with right hemiparesis and aphasia. His past medical history was significant for hypertension, dyslipidemia, and smoking 1 pack per day for many years. Brain MR imaging revealed a small, acute watershed infarct involving the left middle and anterior cerebral artery territories. Intracranial MR angiography suggested a high-grade stenosis involving the left middle cerebral artery (MCA) M1 segment and the left anterior cerebral artery (ACA) Al segment. Before symptom onset, the patient had been on daily aspirin therapy. He was started on intravenous heparin before transfer to our facility. On arrival, diagnostic cerebral angiography confirmed a high-grade flow-limiting stenosis at the left internal carotid artery terminus extending into the proximal middle and anterior cerebral arteries (Fig 1A). While admitted, despite continuous heparin infusion, the patient experienced recurrent TIAs referable to the left anterior circulation.

\section{Procedural Information}

Twenty-four hours before anticipated endovascular therapy, the patient was loaded with $300 \mathrm{mg}$ of clopidogrel by mouth and $81 \mathrm{mg}$ of aspirin. After obtaining informed consent, the patient was placed under general anesthesia. Using standard femoral artery access, a $5 \mathrm{~F}$ guiding sheath was placed in the left internal carotid artery. The patient was fully heparinized, and additional booster doses of heparin were given hourly to maintain an activated clotting time of 250-300 seconds. Initially, a $1.5 \mathrm{~mm} \times 9 \mathrm{~mm}$ Gateway balloon (Boston Scientific) was advanced across the stenosis involving the left ACA segment. The balloon was inflated to a diameter $80 \%$ of the normal native vessel. The balloon was then navigated across the stenosis involving the adjacent left MCA segment and inflated to $80 \%$ of the normal native artery diameter. After the initial angioplasty procedures, the left ACA and MCA stenoses had been eliminated with markedly improved flow dynamics. A $2.5 \mathrm{~mm} \times 15 \mathrm{~mm}$ Wingspan stent (Boston Scientific) was then positioned across the entire length of the left MCA stenosis. Because the stenosis involved the carotid 

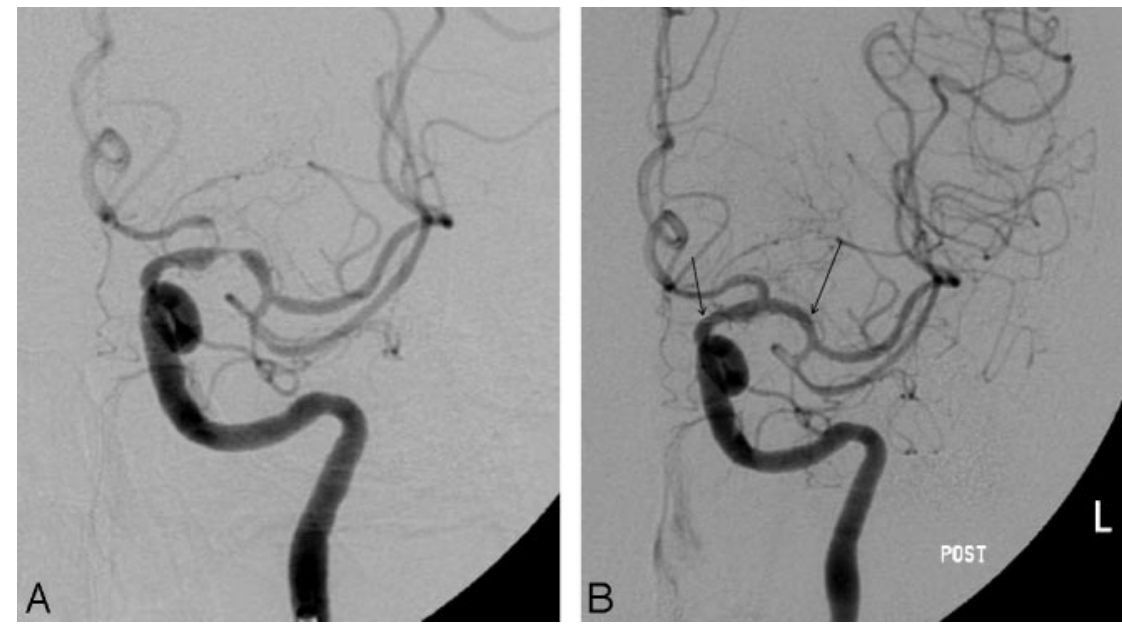

Fig 1. $A$, Left internal carotid artery cerebral angiography demonstrates severe stenoses of the ACA and $\mathrm{MCA}$, which are confluent at the carotid terminus. $B$, After angioplasty alone of the ACA and stent-assisted angioplasty of the MCA, there is normal caliber of the previously stenosed ACA and MCA. Arrows denote the proximal and distal ends of the stent.
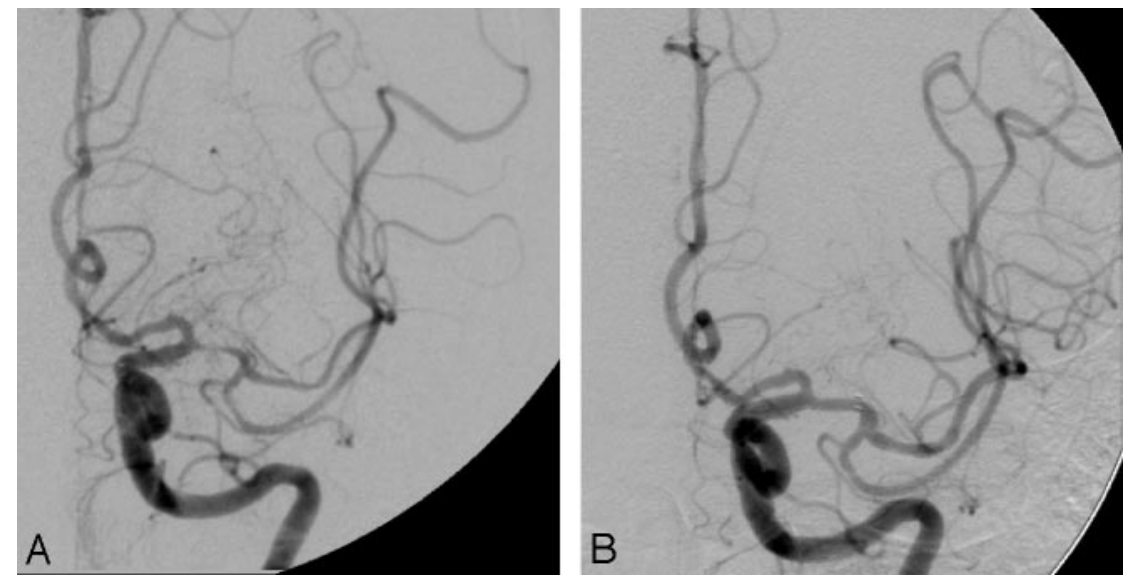

Fig 2. A, Four months after the initial procedure, left internal carotid cerebral angiography demonstrates the ACA to remain normal in caliber. There is a severe recurrent stenosis within the stented segment of the MCA. B, After angioplasty of the severe in-stent restenosis, the left MCA demonstrates markedly improved vessel caliber and improved flow dynamics.

artery terminus, the proximal aspect of the stent was positioned within the supraclinoid internal carotid artery. Cerebral angiography after stent placement demonstrated a normal caliber of the ACA and MCA vessels, without significant residual stenosis (Fig 1B). No local thrombotic or distal embolic events were encountered. The patient awakened from general anesthesia without difficulty and was discharged home 1 week later with mild expressive aphasia and left hemiparesis. He was continued on $81 \mathrm{mg}$ of aspirin daily and $75 \mathrm{mg}$ of clopidogrel daily until follow-up angiography.

Four months after angioplasty and stent placement, the patient returned for a scheduled follow-up cerebral angiogram. His family reported that he had not had any acute neurologic events but that his speech was slowly becoming more incoherent. Left internal carotid angiography demonstrated a normal appearance of the left ACA Al segment that had undergone angioplasty alone. However, there was a severe recurrent stenosis confined to the stented portion of the left MCA (Fig 2A). This resulted in significant hemodynamic compromise to the left MCA territory. Robust left ACA to MCA leptomeningeal collaterals were evident. Balloon angioplasty of the in-stent stenosis resulted in dramatically improved vessel caliber and anterograde flow in the left MCA (Fig 2B). The patient and family noted an immediate improvement in his speech after the angioplasty.

\section{Discussion}

We present this case because the patient's anatomy offers a fortuitous chance to compare, in a single subject, the results of angioplasty alone with the results of stent-assisted angioplasty. Both the M1 and A1 segments initially presented with highgrade stenoses. However, we noted marked neointimal hyperplasia in the stent-bearing M1 segment compared with nearly absent neointimal hyperplasia in the nonstent-bearing A1 segment. This finding implies that the stent itself may elicit neointimal hyperplasia beyond that produced by angioplasty alone. In light of the recent upsurge in enthusiasm for stent placement for ICAD, this case gives us pause concerning what we actually know about the fundamental biology of intracranial vessels in response to stent placement.

There are currently no data from a controlled study comparing angioplasty alone with stent-assisted angioplasty in a large cohort of patients. However, there have been recent reports suggesting high rates of restenosis in stent-assisted angioplasty cases. ${ }^{4-6}$ Subgroup analysis of the same cohort implicated stenoses in young patients in the supraclinoid ICA as those most prone to restenosis. ${ }^{6}$ Given that particular subgroups display a higher propensity to restenosis, it seems that not all stenotic intracranial lesions are alike in their response to stent implantation. Our sin- 
gle case offers some potential advantage over these previous reports, because the nonstented A1 segment acted as an internal control for the stented M1 segment. Of significant note, the patient reported herein was not young, and his restenotic lesion was not in the supraclinoid ICA. This suggests to us that the stent itself may be the primary factor in the development of restenosis. Indeed, a previous series of intracranial angioplasty alone has reported restenosis rates apparently lower than that seen with stent placement. ${ }^{3}$

We readily admit that a single case report can prove nothing definitively. It is true that restenosis is a documented event after angioplasty alone in ICAD. This case and the recent reports of stent-associated restenosis illustrate the critical need for a randomized trial comparing angioplasty alone with stent-assisted angioplasty in patients with ICAD who have failed maximum medical therapy. ${ }^{7}$

\section{References}

1. Chimowitz MI, Lynn MJ, Howlett-Smith H, et al. Comparison of warfarin and aspirin for symptomatic intracranial arterial stenosis. $N$ Engl $\mathrm{J}$ Med 2005;352:1305-16

2. The EC/IC Bypass Study Group. Failure of extracranial-intracranial arterial bypass to reduce the risk of ischemic stroke. Results of an international randomized trial. N Engl J Med 1985;313:1191-200

3. Marks MP, Wojak JC, Al-Ali F, et al. Angioplasty for symptomatic intracranial stenosis: clinical outcome. Stroke 2006;37:1016-20

4. Levy EI, Turk AS, Albuquerque FC, et al. Wingspan in-stent restenosis and thrombosis: incidence, clinical presentation, and management. Neurosurgery 2007;61:644-50

5. Kallmes DF, Cloft HJ. How do we spin Wingspan? AJNR Am J Neuroradiol 2008;29:28-29

6. Turk AS, Levy EI, Albuquerque FC, et al. Influence of patient age and stenosis location on Wingspan in-stent restenosis. AJNR Am J Neuroradiol 2008;29:23-27

7. Kallmes DF, Do HM. Wherefore Wingspan? AJNR Am J Neuroradiol 2007;28: 997-98 